\title{
Packing minor closed families of graphs
}

\author{
Silvia Messuti ${ }^{1}$ \\ Fachbereich Mathematik \\ Universität Hamburg \\ Hamburg, Germany \\ Vojtěch Rödl ${ }^{2,3}$ \\ Department of Mathematics and Computer Science \\ Emory University \\ Atlanta, GA 30322, USA \\ Mathias Schacht ${ }^{4,5}$ \\ Fachbereich Mathematik \\ Universität Hamburg \\ Hamburg, Germany
}

\begin{abstract}
Motivated by a conjecture of Gyárfás, recently Böttcher, Hladký, Piguet, and Taraz showed that every collection $T_{1}, \ldots, T_{n}$ of trees on $n$ vertices with $\sum_{i=1}^{n} e\left(T_{i}\right) \leq\left(\begin{array}{l}n \\ 2\end{array}\right)$ and with bounded maximum degree, can be packed into the complete graph on $(1+o(1)) n$ vertices. We generalize this result where we relax the restriction of packing families of trees to families of graphs of any given non-trivial minor closed class of graphs.
\end{abstract}

Keywords: graph packing, minor closed families, resolvable designs 


\section{Introduction and main result}

A packing of a sequence of graphs $\mathcal{F}=\left(F_{1}, \ldots, F_{n}\right)$ into a graph $H$ is a collection of edge-disjoint subgraphs $H_{1}, \ldots, H_{n} \subseteq H$, such that $H_{i}$ is isomorphic to $F_{i}$ for every $i \in[n]$. A well-known conjecture of Gyárfás [4] states that a sequence of trees $\mathcal{T}=\left(T_{1}, \ldots, T_{n}\right)$, where $v\left(T_{i}\right)=i$ for every $i \in[n]$, packs into $K_{n}$. Note that the sum of the edges over $\mathcal{T}$ is precisely $\left(\begin{array}{l}n \\ 2\end{array}\right)$, hence the packing of $\mathcal{T}$ should use all the edges of $K_{n}$. A restricted approximate version where the host graph is a clique on $(1+o(1)) n$ vertices and the trees have at most $n$ vertices, bounded maximum degree, and the sum over all edges is at most $\left(\begin{array}{l}n \\ 2\end{array}\right)$, was proved by Böttcher, Hladký, Piguet, and Taraz [3]. We extend this result to sequences of graphs from any non-trivial minor closed family.

Theorem 1.1 For any $\varepsilon>0, \Delta \in \mathbb{N}$, and any non-trivial minor closed family $\mathcal{G}$ there exists $n_{0} \in \mathbb{N}$ such that for every $n \geq n_{0}$ the following holds. If $\mathcal{F}=\left(F_{1}, \ldots, F_{n}\right)$ is a sequence of graphs from $\mathcal{G}$, each having order at most $n$ and maximum degree at most $\Delta$, such that $\sum_{i=1}^{n} e\left(F_{i}\right) \leq\left(\begin{array}{l}n \\ 2\end{array}\right)$, then $\mathcal{F}$ packs into $K_{(1+\varepsilon) n}$.

The main idea in the proof is to remove a small separator from each graph, in such a way that all components have small constant size, then pack the components into a large clique contained into $K_{(1+\varepsilon) n}$ and use the remaining vertices for the separators. In fact we prove a more general result and derive Theorem 1.1 from that, and the Separator Theorem of Alon, Seymour, and Thomas [1]. We define a $(\delta, s)$-separable family as a set of graphs each having the property that by removing $\delta$-proportion of the vertices, the resulting components have order at most $s$.

Theorem 1.2 For any $\varepsilon>0$ and $\Delta \in \mathbb{N}$ there exists $\delta>0$ such that for every $s \in \mathbb{N}$ and any $(\delta, s)$-separable family $\mathcal{G}$ there exists $n_{0} \in \mathbb{N}$ such that for every $n \geq n_{0}$ the following holds. If $\mathcal{F}=\left(F_{1}, \ldots, F_{n}\right)$ is a sequence of graphs from $\mathcal{G}$ each having order at most $n$ and maximum degree at most $\Delta$, such that $\sum_{i=1}^{n} e\left(F_{i}\right) \leq\left(\begin{array}{c}n \\ 2\end{array}\right)$, then $\mathcal{F}$ packs into $K_{(1+\varepsilon) n}$.

As mentioned above, Theorem 1.1 easily follows from Theorem 1.2. In fact, the Separator Theorem states that for any minor closed family $\mathcal{G}$ there exists

\footnotetext{
1 Email:silvia.messuti@math.uni-hamburg.de

2 Supported by NSF grant DMS 1301698

3 Email:rodl@mathcs.emory.edu

4 Supported through the Heisenberg-Programme of the Deutsche Forschungsgemeinschaft (DFG Grant SCHA 1263/4-2)

5 Email:schacht@math.uni-hamburg.de
} 
a constant $c_{\mathcal{G}}$ such that a graph $G \in \mathcal{G}$ of order $n$ has a separator of size at most $c_{\mathcal{G}} n^{1 / 2}$ and the components have order at most $n / 2$. Applying such result recursively to each component, for $i>0$ iterations, leads to a separator $U \subseteq V(G)$ such that

$$
|U| \leq c_{\mathcal{G}} n^{1 / 2}+\cdots+2^{i-1} c_{\mathcal{G}}\left(\frac{n}{2^{i-1}}\right)^{1 / 2}=c_{\mathcal{G}} n^{1 / 2} \frac{\sqrt{2}^{i}-1}{\sqrt{2}-1}<3 c_{\mathcal{G}} n^{1 / 2} 2^{i / 2}
$$

and each component of $G-U$ has order at most $n / 2^{i}$. For given $\delta>0$ we can apply this with $i=\left\lfloor 2 \log _{2}\left(\delta n^{1 / 2} / 3 c\right)\right\rfloor$ and obtain a separator $U$ of size at most $\delta n$, and a set of components all of which have order at most $18 c_{\mathcal{G}}^{2} / \delta^{2}$. This shows that the minor-closed family $\mathcal{G}$ is $\left(\delta, 18 c_{\mathcal{G}}^{2} / \delta^{2}\right)$-separable for any $\delta>0$. Hence, we can apply Theorem 1.2, and Theorem 1.1 follows.

\section{Sketch of the proof}

We provide a sketch of the proof of Theorem 1.2 in which we ignore the choice of the parameters and focus on the construction of the packing. We assume that for $\varepsilon$ and $\Delta$ we are given a sequence $\mathcal{F}$ (from some appropriate $(\delta, s)$ separable family $\mathcal{G}$ ) that satisfies the assumptions of the theorem, and that $n$ is sufficiently large. We consider each graph $F_{i}$ of the sequence together with a fixed $(\delta, s)$-separation $\left(U_{i}, \mathcal{C}_{i}\right)$, where $U_{i} \subseteq V\left(F_{i}\right)$ is the separator and has size $\left|U_{i}\right| \leq \delta v\left(F_{i}\right)$, and $\mathcal{C}_{i}$ is the component graph, i.e., the subgraph of $F_{i}$ induced on $V\left(F_{i}\right) \backslash U_{i}$, where each component $C$ has order at most $s$. We define the boundary $\partial C$ of a component $C$ as the subset of the vertices that are adjacent to the separator, and more generally the boundary of a set of components as the union of the boundary sets of all its elements.

The vertices of the host graph $K_{(1+\varepsilon) n}$ will be split into a large part $X$ of order $(1+\xi) n$ for some carefully chosen $\xi=\xi(\varepsilon, \Delta)>0$, and a small part $Y=V \backslash X$. We will pack the component graphs $\left\{\mathcal{C}_{i}\right\}_{i \in[n]}$ into the clique $K_{X}$ spanned on $X$, the sets $\left\{U_{i}\right\}_{i \in[n]}$ into the clique $K_{Y}$ spanned on $Y$, and use the edges of the complete bipartite graph $K_{X, Y}$ induced by $X$ and $Y$ for the necessary connections. Since every edge of $K_{X, Y}$ can be used only once in the packing, we will make sure that each vertex of $X$ will only host a few vertices from the boundary of $\mathcal{F}$.

\subsection{Packing the component graphs}

The packing of $\left\{\mathcal{C}_{i}\right\}_{i \in[n]}$ consists of two steps: the assignment and the embedding. In the assignment phase we group the components by isomorphism 
types, decompose $K_{X}$ into such graphs, and assign each component to one of its copies in the decomposition. In the embedding phase we actually "place" the copies of the components somewhere into $K_{X}$, i.e., we assign the copies of the components to the vertices of $K_{X}$. We will do this by randomly choosing a permutation of the vertices, and show that with positive probability the resulting packing is balanced in the sense that no vertex of $K_{X}$ hosts too many boundary vertices.

The main idea in the assignment phase is to decompose $K_{X}$ into smaller graphs isomorphic to the components in $\left\{\mathcal{C}_{i}\right\}_{i \in[n]}$ and assign each component to one of its copies in the decomposition. However, two components from the same graph must be mapped vertex disjointly by definition, so we need a decomposition that can be partitioned into (preferable perfect) matchings. For that we apply a result by Ray-Chaudhuri and Wilson [5] that states that for $m \geq 2$ and sufficiently large $n$, if the necessary divisibility conditions are met, then there exists a resolvable $K_{m}$-decomposition of $K_{n}$, i.e., a decomposition that can be partitioned into $K_{m}$-factors. For general $G$, resolvable decompositions do not necessarily exist. However, due to Alon and Yuster [2], we can decompose a $K_{n}$ into $G$ in such a way that almost all matchings contain a large number of copies of $G$, if the divisibility conditions are satisfied and $n$ is sufficiently large.

Let $\mathcal{S}=\left\{S_{1}, \ldots, S_{\sigma}\right\}$ be the set of graphs on at most $s$ vertices and maximum degree at most $\Delta$ isomorphic to some component in $\left\{\mathcal{C}_{i}\right\}_{i \in[n]}$, with $e\left(S_{1}\right) \geq \cdots \geq e\left(S_{\sigma}\right)$. We assume that $X$ has size $(1+\xi) n$ for some appropriately chosen $\xi$. We will decompose a subgraph $K_{N}$ of $K_{X}$ with $\left(1+\frac{\xi}{2}\right) n \leq N \leq(1+\xi) n$ such that there exist $m$ and $\ell$ with $s \ll \ell \ll m \ll N$, for which $K_{N}$ has a resolvable $K_{m}$-decomposition $\mathcal{D}^{m, N}, K_{m}$ has a resolvable $K_{\ell}$-decomposition $\mathcal{D}^{\ell, m}$, and $K_{\ell}$ can be decomposed into $S$ in such a way that at least $(1-\zeta) \frac{(\ell-1) v(S)}{2 e(S)}$ matchings of the decomposition $\mathcal{D}^{S, \ell}$ contain at least $(1-\zeta) \ell / v(S)$ copies of $S$, where $S$ is any graph from $\mathcal{S}$ and $\zeta$ is an appropriately chosen constant.

We shall pack the component graphs $\left\{\mathcal{C}_{i}\right\}_{i \in[n]}$ into $K_{N}$ one by one in such a way that each $\mathcal{C}_{i}$ is completely contained in one of the $K_{m}$-factors of $K_{N}$. Roughly speaking, for each $\mathcal{C}_{i}$ we group the components by isomorphism types, and for every such isomorphism type $S \in \mathcal{S}$ we allocate an $S$-matching in sufficiently many copies of $K_{m}$. Since for different graphs in $\left\{\mathcal{C}_{i}\right\}_{i \in[n]}$ we may need to use the same $K_{m}$ for hosting different graphs from $\mathcal{S}$, we decompose $K_{m}$ into copies of $K_{\ell}$. This way, given different $S$ and $S^{\prime}$ from $\mathcal{S}$, we may allocate an $S$-matching in $K_{m}$ by using an $S$-decomposition into one $K_{\ell}$-factor, and an $S^{\prime}$-matching in the same $K_{m}$ by using an $S^{\prime}$-decomposition in a different $K_{\ell^{-}}$ 
factor. We proceed this way until essentially all edges of the given $K_{m}$-factor are used. At this point we switch to the next $K_{m}$-factor in $K_{N}$ and proceed the same way. In order to keep the amount of unused edges small in such a change of $K_{m}$-factors, we have to make sure that all $K_{m}$ 's in such a factor are populated by component graphs $\mathcal{C}_{i}$ in a balanced way. This will be achieved by choosing the copies of $K_{m}$ for each $\mathcal{C}_{i}$ among those that have the least number of used edges. We now describe the assignment of the components of a graph in detail.

For the set of components $\mathcal{C}_{i}$ of $F_{i}$ and for a graph $S \in \mathcal{S}$, we define $M_{i}(S)$ as the number of copies of $K_{m}$ we shall reserve for embedding all the components isomorphic to $S$ from $\mathcal{C}_{i}$ into $K_{N}$. Note that we ignore the $S$-matchings that contain less than $(1-\zeta) \frac{\ell}{v(S)}$ copies of $S$. We assign the components of $\mathcal{C}_{i}$ to the current $K_{m}$-factor, in which the $K_{m}$ 's are considered to be ordered starting from the one in which the least number of edges has been allocated to some of the previous component graphs. Moreover, in each $K_{m}$ we have for every $S \in \mathcal{S}$ a current $K_{\ell}$-factor in which all $K_{\ell}$ 's are $S$-decomposed. We assign the components of $\mathcal{C}_{i}$ to $M_{i}=\sum_{s=1}^{\sigma} M_{i}\left(S_{s}\right)$ of these $K_{\ell}$-factors each from a different $K_{m}$ as described below.

We start by picking the first $M_{i}$ copies of $K_{m}$ in the current $K_{m}$-factor and assign each of them a graph from $\mathcal{S}$ isomorphic to some component of $\mathcal{C}_{i}$. Recall that the $K_{m}$ 's are ordered from the one with the most number of available edges to that with the most number of used edges, and the graphs in $\mathcal{S}$ are ordered from the densest to the sparsest. Hence, by assigning $S_{1}$ to the first $M_{i}\left(S_{1}\right)$ copies of $K_{m}, S_{2}$ to the next $M_{i}\left(S_{2}\right)$ copies, and so on, we maintain an "approximately balanced" assignment to the current $K_{m}$-factor. Once we decide which $S$ goes to which $K_{m}$, we look at the current $S$-decomposed $K_{\ell^{-}}$ factor in the respective $K_{m}$ 's and allocate a large $S$-matching to which we assign the components from $\mathcal{C}_{i}$ that are isomorphic to $S$.

Before we repeat the procedure for $\mathcal{C}_{i+1}$, we update our workspace as follows. For every $K_{\ell}$-factor we just used for the components from $\mathcal{C}_{i}$ isomorphic to some $S$ we check whether it contains another large $S$-matching that has not been allocated yet. Note that each time we consider a $K_{\ell}$-factor for the assignment we use all the $K_{\ell}$ 's contained in it, hence the $S$-matchings get exhausted in all the $K_{\ell}$ 's at the same moment. If all the large $S$-matchings have been used, then we pick a new $K_{\ell}$-factor, if available, in the same $K_{m}$. To each newly selected $K_{\ell}$ we apply $\mathcal{D}^{S, \ell}$, and this becomes the current $K_{\ell}$-factor for $S$ in this $K_{m}$. If we are in the situation that there is no available $K_{\ell^{-}}$ factor in at least one of the $K_{m}$ 's, then we consider the $K_{m}$ 's of this $K_{m}$-factor in $K_{N}$ as completely used, and we will show that the "balancing procedure" 
described above ensures that most $K_{m}$ 's are "almost full". At this point we replace our current $K_{m}$-factor with a new one, and in each $K_{m}$ we set up current $K_{\ell}$-factors for each $S \in \mathcal{S}$.

The procedure above produces an assignment of the components in $\left\{\mathcal{C}_{i}\right\}_{i \in[n]}$ to their copies inside $K_{N}$ such that all components from each $\mathcal{C}_{i}$ are vertex disjoint. It is left to show that there is enough room for all the component graphs, i.e., that the edges we waste in the assignment procedure plus those contained into the $S$-matchings that were not used because they contained too few copies of $S$ are fewer than the additional edges we allow by considering $K_{N}$ instead of $K_{n}$. In order to determine the number of edges wasted in the assignment procedure, observe that a $K_{m}$ is considered to be full when for some $S$ there is no large $S$-matching available in the current $K_{\ell}$-factor and all other $K_{\ell}$-factors in that $K_{m}$ have been used, or are currently reserved for some other $S^{\prime} \in \mathcal{S}$. At this moment, in the worst case all current $K_{\ell}$-factors are empty, hence the waste is at most $\sigma \frac{m}{\ell}\left(\begin{array}{l}\ell \\ 2\end{array}\right)$. Since we pick the least used $K_{m}$ 's, we may assume that in every other $K_{m}$ of the current $K_{m}$-factor we have the same waste, yielding a total waste of $\frac{N}{m} \sigma \frac{m}{\ell}\left(\begin{array}{l}\ell \\ 2\end{array}\right)$ pairs in the $K_{m}$-factor, and hence $\sigma \frac{\ell-1}{m-1}\left(\begin{array}{c}N \\ 2\end{array}\right)$ edges in the whole $K_{N}$. As for the edges contained in small $S$ matchings, one can show that their total number is at most $\left(\zeta-\zeta^{2}\right)\left(\begin{array}{c}N \\ 2\end{array}\right)$. By our choice of $m$ and $\zeta$ we have that the total number of unused edges in $K_{N}$

$$
\sigma \frac{\ell-1}{m-1}\left(\begin{array}{c}
N \\
2
\end{array}\right)+\left(\zeta-\zeta^{2}\right)\left(\begin{array}{c}
N \\
2
\end{array}\right)
$$

is smaller than $\left(\begin{array}{c}N \\ 2\end{array}\right)-\left(\begin{array}{l}n \\ 2\end{array}\right)$. This shows that all component graphs in $\mathcal{F}$ fit into $K_{N}$.

For the actual embedding, i.e., the assignment of the copies of the components to the vertices of $K_{N}$, we have to make sure that each vertex of $K_{N}$ hosts at most $\xi n$ vertices from the boundary of $\mathcal{F}$. For that we will first pick a random permutation of the $K_{m}$ 's in each $K_{m}$-factor, and then a random permutation of the vertices inside each $K_{m}$.

We define a labelling of the $K_{m}$ 's in the assignment by assigning each $K_{m}$ the (unordered) sequence of the boundary degrees of its vertices, i.e., for each vertex in the $K_{m}$ we count how many times it was used for the embedding of a boundary vertex. For some appropriately chosen constant $\eta$ we say that a label is common if at least $\frac{\eta}{2^{m}} \frac{N(N-1)}{m(m-1)} K_{m}$ 's have that label and rare otherwise. We shall also use $\eta$ as the multiplicative error in the following applications of Chernoff's inequality.

Given the $K_{m}$-decomposition of $K_{N}$, we look at one vertex $v$ and choose 
the $K_{m}$ 's incident to $v$ by randomly picking one $K_{m}$ in each $K_{m}$-factor. Some applications of Chernoff's inequality show that with positive probability, for all vertices we have that the occurrences of every common label among the $K_{m}$ 's incident to each vertex roughly agree in proportion with the occurrences of that label in the decomposition. Since all but at most an $\eta$-proportion of the $K_{m}$ 's in the decomposition have a common label, we obtain that the number of $K_{m}$ 's with common labels attached to each vertex $v$ is at least $(1-\eta)^{2} \frac{N-1}{m-1}$, and the number of $K_{m}$ 's with rare labels for $v$ is at most $2 \eta \frac{N-1}{m-1}$.

Next we shall also permute the vertices inside each $K_{m}$ so that the boundary degrees in each $K_{m}$ will be equally distributed. For a vertex $v$ and a common label $A$ we pick a random value in the boundary degree sequence of each $K_{m}$ labelled with $A$ and by applying Chernoff's inequality we can show that the sum of boundary degrees of $v$ in all these $K_{m}$ 's is concentrated around its expectation. By summing over all common labels, we have that with positive probability there exists a permutation for which all vertices have boundary degree at most $(1+\eta)^{2} \frac{1}{N} \frac{\xi}{2} n^{2}$. By adding the largest possible boundary degree, i.e., $m$ for at most $2 \eta \frac{N-1}{m-1} K_{m}$ 's with rare labels, we obtain that each vertex of $K_{N}$ hosts at most

$$
(1+\eta)^{2} \frac{1}{N} \frac{\xi}{2} n^{2}+2 \eta \frac{N-1}{m-1} m<\left(\frac{(1+\eta)^{2}}{1+\xi / 2} \frac{\xi}{2}+2 \eta(1+\xi)\right) n<\xi n
$$

vertices from the boundary of $\mathcal{F}$.

\subsection{Packing the separators}

Having constructed a packing of the components where each vertex of $X$ hosts at most $\xi n$ vertices from the boundary of $\mathcal{F}$, it is left to show that we can use just a few more vertices to embed the separators. In order to obtain a packing of $\mathcal{F}$ we have to satisfy the following conditions:

(i) for every $i \in[n]$, the vertices of $U_{i}$ have to be mapped injectively into $Y$;

(ii) each edge in $K_{X, Y}$ can be used at most once;

(iii) each edge in $K_{Y}$ can be used at most once.

We embed the separators one by one, vertex by vertex. In the following we describe the embedding of an arbitrary vertex $u$ from an arbitrary separator $U_{i}$ We assume that all vertices of $U_{j}$ with $j<i$ and up to at most $\left|U_{i}\right| \leq \delta n$ vertices of $U_{i}$ were already embedded, and that so far we made sure that every vertex in $Y$ was used at most $3 \frac{\delta n^{2}}{|Y|}$ times. We will embed $u$ in such a way that the constraints discussed above will be obeyed, and afterwards each 
vertex of $Y$ is still used at most $3 \frac{\delta n^{2}}{|Y|}$ times. For that we collect the restrictions given by the previous embeddings.

(i) Since the vertices of $U_{i}$ have to be embedded injectively into $Y$, up to at most $\left|U_{i}\right| \leq \delta n$ vertices in $Y$ might be forbidden for the embedding of $u$.

(ii) Every edge in $K_{X, Y}$ can be used at most once, hence we have to embed $u$ into a vertex $y$ such that all edges between $y$ and the vertices $X^{\prime}$ of $X$ that embed the neighbours of $u$ in $\mathcal{C}_{i}$ are available. Since $u$ has at most $\Delta$ neighbours in $\mathcal{C}_{i}$, and each vertex in $X^{\prime}$ hosts at most $\xi n$ boundary vertices, each having at most $\Delta$ neighbours in $\left\{U_{i}\right\}_{i \in[n]}$, at most $\Delta^{2} \xi n$ more vertices might be forbidden.

(iii) Also the edges in $K_{Y}$ can be used at most once. This means that the target vertex for $u$ must be chosen in such a way that all the edges to the vertices $Y^{\prime}$ that embed its neighbourhood in $U_{i}$ have not been used yet. Note that each vertex in $Y^{\prime}$ may already host up to $3 \frac{\delta n^{2}}{|Y|}$ vertices from $\left\{U_{i}\right\}_{i \in[n]}$, hence the embeddings of the neighbours of such vertices are also forbidden for the choice of $u$. Since $u$ has at most $\Delta$ neighbours in $U_{i}$, and each vertex embedded into $Y^{\prime}$ has at most $\Delta$ neighbours in $Y$, this results in excluding at most $\Delta^{2} 3 \frac{\delta n^{2}}{|Y|}$ additional vertices from $Y$.

An appropriate choice of the constants ensures that the set of candidates for the embedding of $u$ is larger than $|Y| / 2$. Since we have to embed at most $\sum_{i \in[n]}\left|U_{i}\right| \leq \delta n^{2}$ vertices in total, some vertex $y \in Y$ was used at most

$$
\frac{\delta n^{2}}{|Y| / 2}<3 \frac{\delta n^{2}}{|Y|}-1
$$

times, and this vertex we choose for the embedding of $u$. We have thus shown that for each vertex of $\left\{U_{i}\right\}_{i \in[n]}$ we can pick a vertex in $Y$ such that all the edges needed for the necessary connections are available, and hence obtain a packing of $\mathcal{F}$.

\section{References}

[1] N. Alon, P. Seymour, and R. Thomas, A separator theorem for nonplanar graphs, J. Amer. Math. Soc. 3 (1990), no. 4, 801-808.

$[2]$ N. Alon and R. Yuster, Every $H$-decomposition of $K_{n}$ has a nearly resolvable alternative, European J. Combin. 21 (2000), no. 7, 839-845. 
[3] J. Böttcher, J. Hladký, D. Piguet, and A. Taraz, An approximate version of the tree packing conjecture, manuscript.

[4] A. Gyárfás and J. Lehel, Packing trees of different order into $K_{n}$, Combinatorics (Proc. Fifth Hungarian Colloq., Keszthely, 1976), Colloq. Math. Soc. János Bolyai, vol. 18, North-Holland, Amsterdam, 1978, pp. 463-469.

[5] D. K. Ray-Chaudhuri and R. M. Wilson, The existence of resolvable block designs, Survey of combinatorial theory (Proc. Internat. Sympos., Colorado State Univ., Fort Collins, Colo., 1971), North-Holland, Amsterdam, 1973, pp. 361-375. 